\title{
No hay consenso sobre la forma de ajustar las dosis farmacológicas según la función renal
}

\begin{abstract}
Objetivo
Comparar la dosis recomendada por cuatro reconocidas fuentes de información fármaco-terapéutica respecto del ajuste de la dosis de drogas de uso frecuente a la función renal del paciente; y comparar la definición de insuficiencia renal utilizada, así como el aval bibliográfico de las recomendaciones brindadas en cada una de las fuentes consultadas.
\end{abstract}

\section{Fuente y selección de datos}

En forma sistemática e independiente, los autores obtuvieron de cuatro fuentes de información fármaco-terapéutica la recomendación acerca del ajuste de dosis a la función renal para 100 fármacos de uso frecuente en su hospital. Las fuentes de datos en cuestión fueron: British Nacional Formulary, Martindale: the Complete Drug Reference, American Hospital Formulary System Drug Information, Drug Prescribing in Renal Failure.

\section{Resultados principales}

Las cuatro bases difirieron en su definición de insuficiencia renal: dos de ellas hicieron una definición cualitativa utilizando rótulos como "deterioro renal" o "insuficiencia renal" pero sin profundizar en su definición, mientras que las otras dos dieron una definición cuantitativa, dividiendo dicho síndrome en grados representados por distintos rangos de filtrado glomerular. Sin embargo los rangos considerados fueron diferentes entre ambas bases. Una consideró más de 50,50 a 20,20 a 10 y menos de $10 \mathrm{~mL} / \mathrm{min}$; y la otra, más de 50,50 a 10 , y menos de $10 \mathrm{~mL} / \mathrm{min}$.

Las fuentes difirieron también en la recomendación del ajuste de las dosis a la insuficiencia renal. De las 100 drogas analizadas, cada una de las bases recomendó ajuste de dosis en dicha situación en el $54 \%, 62 \%, 56 \%$ y $53 \%$ de los fármacos respectivamente. Incluso se observó que, en seis drogas, mientras una fuente recomendaba no ajustar la dosis en los pacientes con insuficiencia renal, otra contraindicaba su uso. Fue llamativa, en todas las fuentes, el escasa respaldo bibliográfico de los fundamentos en que se basaban las diferentes recomendaciones.

\section{Conclusiones}

Las cuatro fuentes de información fármaco-terapéutica analizadas difirieron en algún grado en su definición de insuficiencia renal y en la recomendación de ajuste de dosis a la función renal de los fármacos. En todas las fuentes hubo escasa presentación de la evidencia en la que se basaban las recomendaciones emitidas.

Fuente de financiamiento: Framework, AMIKA. Sin declaración de conflictos de intereses.

\section{Comentario}

Este artículo pone en evidencia una realidad: no hay aun unanimidad en la definición de insuficiencia renal, ni en los esquemas de ajuste de dosis de fármacos a la función renal'. Este hecho explica la disparidad en las recomendaciones dadas por las cuatro fuentes de información fármaco-terapéutica evaluadas por este trabajo. El panorama es aun más incierto en situaciones clínicas como la insuficiencia hepática o la vejez, en las cuales si bien es sabido que hay cambios fisiológicos y farmacocinéticos que hacen necesario un ajuste de dosis, se carece muchas veces de tablas o esquemas destinados a tal fin, similares a los existentes para la insuficiencia renal $^{2}$
Definitivamente, las fuentes de consulta fármaco-terapéutica deberían presentar la evidencia y el grado de la misma en que se basan cada una de sus recomendaciones.

Dada la variabilidad existente en las recomendaciones de dosificación, lo ideal es elegir una fuente de información confiable y guiarse siempre por ella. Si llegara a presentase una situación clínica en la que una medicación debiera ser indefectiblemente utilizada y no se hallara información clara en cuanto a su ajuste de dosis, sería de gran ayuda para su correcta aplicación el conocimiento de la farmacocinética de la droga en cuestión así como el diálogo con el especialista en la materia.

Carlos Musso [ Médico Especialista en Nefrología. Servicio de Nefrología del Hospital Italiano de Buenos Aires. ]

Musso C. No hay consenso sobre la forma de ajustar las dosis farmacológicas según la función renal. Evid. actual. páct. ambul. 9(3);70. May-Jun. 2006. Comentado de: Vidal L, Shavit M, Fraser A, y col. Systematic comparison of four sources of drug information regarding adjustment of dose for renal function. BMJ 2005:331:263. PMID: 15905228.

Referencias

1.Liaño F, Pascual J. Insuficiencia renal aguda. 2000, Barcelona. Masson. Lacy C, Armstrong L.

2. Goldman M, Lance L. Drug information handbook internacional. 2005, Ohio. Lexi-Comp.

INFO - EVIDENCIA

¿Qué es Evidencia, Actualización en la Práctica Ambulatoria?

Una publicación independiente editada cada dos meses desde 1997 por la Fundación MF, Organización sin fines de lucro dedicada a promover el desarrollo de la medicina familiar y la atención primaria de la salud.

¿Cuál es el objetivo de Evidencia?

Contribuir a la educación continua y a la actualización de los profesionales de la salud de la región en el área de la atención ambulatoria.

\section{¿Contenidos de Evidencia}

- Información independiente, actualizada y resumida en forma sencilla y en castellano sobre temas relevantes de la atención ambulatoria.

- Artículos seleccionados por su calidad y relevancia clínica, resumidos y comentados críticamente por destacados profesionales del área.

- Revisiones sobre temas clínicos, epidemiológicos, sanitarios o humanísticos, de importancia para la práctica ambulatoria.

Notas farmacológicas para la actualización rápida de temas de terapéutica clínica.

Discusión de casos clínicos con herramientas de Medicina Basada en la Evidencia.

Glosario de términos de epidemiología y medicina basada en la evidencia

¿Cómo es posible acceder a Evidencia?

Por suscripción a la edición en papel, o bien por Intenet, en nuestro sitio:

http://www.evidencia.org 